\title{
Capacity Performance Analysis of M-ary PPM TH-UWB Systems in the Presence of Narrowband Interference
}

\author{
Nikos V. Kokkalis, P. Takis Mathiopoulos, George K. Karagiannidis, and Christos S. Koukourlis
}

\begin{abstract}
The capacity of ultra-wideband (UWB) systems in presence of narrowband interference (NBI) is studied. By appropriately modifying the Shannon capacity formula, an analytical expression for the capacity of $M$-ary pulse position modulation (PPM) signals in the presence of NBI is obtained. Performance evaluation results for the capacity of such signals as a function of the NBI carrier frequency and power are also presented.
\end{abstract}

Index Terms: Capacity, narrowband interference (NBI), pulse position modulation (PPM), spread spectrum communication, time hopping, ultra-wideband (UWB).

\section{INTRODUCTION}

Ultra-wideband (UWB) radio system technology plays an important role in current research for wireless personal area networks [1]. One of the most widely studied schemes for such systems utilizes $M$-ary pulse position modulation (PPM) combined with time-hopping (TH) as a multiple access technique [2], [3]. The multiple access performance of this scheme has been extensively studied in the past [4]-[8]. Its performance in a multipath fading environment has also been addressed [9], [10].

The performance of UWB systems in channels with narrowband interference (NBI) has been studied in [12] and [13] by evaluating the processing gain of the single user correlator receiver, which is optimal in an additive white Gaussian noise (AWGN) environment. Further interference studies have shown that the single user receiver does not effectively combat strong NBI, especially for high data rate UWB systems where the spreading factor is relatively small [10]. In the same reference, a minimum mean square error (MMSE) receiver which improves the performance of such systems has been proposed and analyzed. These UWB-NBI studies employ as performance measures the processing gain [12], [13] and bit error rate [10].

The problem of estimating the capacity of UWB systems has received relatively less attention. It has been studied for the AWGN channel in [5] and [6]. A more recent study [11] evaluates the cut-off rate, also known as the practical capacity, of such systems in the presence of multiple access interference. Our current contribution focuses on studying the Shannon capacity of

Manuscript received August 29, 2006; approved for publication by Jinho Choi, Division II Editor, January 11, 2008.

N. V. Kokkalis and C. S. Koukourlis are with the Department of Electrical and Computer Engineering, Democritus University of Thrace, 12 Vas. Sofias Street, GR67100 Xanthi, Greece, email: \{nkokkali, ckoukou\} @ee.duth.gr.

P. T. Mathiopoulos is with the Institute for Space Applications \& Remote Sensing, National Observatory of Athens, Metaxa \& Vas. Pavlou Street, Palea Penteli, GR15236 Athens, Greece, email: mathio@space.noa.gr.

G. K. Karagiannidis is with the Department of Electrical and Computer Engineering, Aristotle University of Thessaloniki, GR54124 Thessaloniki, Greece, email: geokarag@auth.gr. such systems in the presence of NBI. As well known, capacity is considered as a more fundamental performance measure since it provides limits on achievable performance. Like other capacity studies [6]-[11], the effect of multipath fading is not considered in this paper.

The remainder of this paper is organized as follows. In Section II the UWB and NBI signal models are presented and in Section III a method for evaluating the capacity is proposed and analyzed. Various performance evaluation results are presented and discussed in Section IV.

\section{SYSTEM MODEL}

A typical $M$-ary PPM TH-UWB transmitted signal is mathematically represented as [2]:

$$
s(t)=\sum_{i} \sqrt{E_{p}} q\left(t-i T_{f}-c_{i} T_{c}-\delta_{m}\right), 1 \leq m \leq M
$$

where $q(t)$ is the UWB pulse, a Gaussian monocycle with duration $T_{p}$. In the above equation $E_{p}$ is the received energy per pulse, $T_{f}$ is the pulse repetition interval, usually referred to as frame, $c_{i}$ is the TH sequence, $T_{c}$ the TH slot duration, and $\delta_{m}$ is the modulation shift for $M$-ary orthogonal PPM $\left(\delta_{m}-\delta_{m-1} \geq\right.$ $\left.T_{p}\right)$. The pulse power is $P_{p}=E_{p} / T_{p}$. The TH sequence is modeled as a series of independent uniformly distributed random numbers taking integer values in the range $\left[0, T_{f} / T_{c}\right]$. Assuming $N_{p}$ pulses per symbol are transmitted, the symbol rate is $R_{s}=1 /\left(N_{p} T_{f}\right)$ and thus the symbol duration is $T_{s}=N_{p} T_{f}$. The spreading factor is $\beta=T_{f} / T_{p}$, the pulse rate, $\lambda=1 / T_{f}$, is the average rate of the transmitted pulses and the effective bandwidth ( $3 \mathrm{~dB}$ bandwidth) is $W_{p}=1.297 / T_{p}$ [12].

The NBI waveform, $j(t)$, is modeled as a phase and/or amplitude modulated signal, e.g., phase shift keying (PSK) or quadrature amplitude modulation (QAM), with an ideal block spectrum of bandwidth $W_{J}$, i.e.,

$$
\begin{aligned}
j(t) & =\sum_{i} w_{n}\left(t-i T_{J}\right)=\sqrt{J_{0}} \sum_{i} \frac{\sin \left[\pi W_{J}\left(t-i T_{J}\right)\right]}{\pi\left(t-i T_{J}\right)} \\
& \times\left\{a_{n} \cos \left[2 \pi f_{c}\left(t-i T_{J}\right)\right]+b_{n} \sin \left[2 \pi f_{c}\left(t-i T_{J}\right)\right]\right\}
\end{aligned}
$$

where $J_{0}$ is the power spectral density (PSD) of the interfering signal received by the UWB receiver, $f_{c}$ its carrier frequency, $T_{J}$ the symbol duration, and $w_{n}(t)$ and $\left\{a_{n}, b_{n}\right\}$ are the waveform and the in-phase and quadrature components of the $n$th signal taking values according to the specific modulation used with $1 \leq n \leq M_{J}$ where $M_{J}$ is the number of modulated signals. The NBI power is $P_{J}=J_{0} W_{J}$.

Assuming an AWGN channel and a single NBI source the received signal can be expressed as:

$$
r(t)=s\left(t-\tau_{s}\right)+j\left(t-\tau_{j}\right)+n(t)
$$


where $n(t)$ is the AWGN waveform with zero mean and one sided PSD $N_{0}$ and the delays $\tau_{s}$ and $\tau_{j}$ are due to the asynchronous transmission.

A typical UWB receiver consists of $M$ correlators matched to the template functions $u_{i}(t)=q\left(t-\delta_{i}\right), 1 \leq i \leq M$. Assuming that the receiver is perfectly synchronized with the UWB signal, the output signal of the $\nu$ th correlator at the $k$ th symbol can be expressed as:

$$
\begin{aligned}
y_{k, \nu}= & \sum_{\kappa=k N_{p}}^{(k+1) N_{p}-1}\left\{\int_{\kappa T_{f}}^{(\kappa+1) T_{f}} s(t) u_{\nu}\left(t-\kappa T_{f}-c_{\kappa} T_{c}\right) d t+\right. \\
& \int_{\kappa T_{f}}^{(\kappa+1) T_{f}} j\left(t-\tau_{j}+\tau_{s}\right) u_{\nu}\left(t-\kappa T_{f}-c_{\kappa} T_{c}\right) d t+ \\
& \left.\int_{\kappa T_{f}}^{(\kappa+1) T_{f}} n\left(t+\tau_{s}\right) u_{\nu}\left(t-\kappa T_{f}-c_{\kappa} T_{c}\right) d t\right\} \\
= & \sum_{\kappa=k N_{p}}^{(k+1) N_{p}-1}\left[X_{\kappa, \nu}+I_{\kappa, \nu}+N\right]
\end{aligned}
$$

where $X_{\kappa, \nu}, I_{\kappa, \nu}$, and $N$ are the contributions of the UWB, NBI and AWGN signals, respectively. The UWB signal term is $X_{\kappa, \nu}=\sqrt{E_{p}}$ if $\nu=m$ and zero otherwise, while the noise term remains Gaussian with variance $\sigma_{N}^{2}=N_{0}$. Since the template functions in (4) are non-zero for a small fraction of the integration interval, for the convenience of the analysis this integration limits can be extended to $(-\infty,+\infty)$. It is also convenient to express $I_{\kappa, \nu}$ as:

$$
\begin{gathered}
I_{\kappa, \nu}=\sum_{i} I_{\kappa, \nu}^{i, w_{n}}, \\
I_{\kappa, \nu}^{i, w_{n}}=\int_{-\infty}^{+\infty} w_{n}\left(t-i T_{J}-\tau_{j}+\tau_{s}\right) u_{\nu}\left(t-\kappa T_{f}-c_{\kappa} T_{c}\right) d t .
\end{gathered}
$$

Since $w_{n}(t)$ has a block spectrum with bandwidth $W_{J}$, by using the Fourier transform, $I_{\kappa, \nu}^{i, w_{n}}$ can be expressed as:

$$
I_{\kappa, \nu}^{i, w_{n}}=\sqrt{J_{0}} r_{n} \int_{f_{c}-W_{J} / 2}^{f_{c}+W_{J} / 2} Q(f) \exp \left[j\left(2 \pi f \tau+\theta_{n}\right)\right] d f
$$

where $\tau=\kappa T_{f}+c_{\kappa} T_{c}-i T_{J}-\tau_{j}+\tau_{s}, Q(f)$ is the Fourier transform of $q(t)$, and $\left\{r_{n}, \theta_{n}\right\}$ is the polar form of the coefficients $\left\{a_{n}, b_{n}\right\}$. Since $W_{J} \ll W_{p}$, (6) simplifies to:

$I_{\kappa, \nu}^{i, w_{n}}=\sqrt{J_{0}} r_{n} Q\left(f_{c}\right) \frac{\sin \left(\pi W_{J} \tau\right)}{\pi \tau} \cos \left(2 \pi f_{c} \pi W_{J} \tau+\theta_{n}\right)$.

\section{CAPACITY EVALUATION}

It is mathematically convenient to express the transmitted and received signals on a certain time interval as vectors. Thus, a single $M$-ary PPM UWB pulse can be represented as a vector, $\mathbf{x}_{\mathbf{k}, \mathbf{m}}=\left[x_{k, 1}, x_{k, 2}, \cdots, x_{k, M}\right]^{\mathrm{T}}, 1 \leq m \leq M$, where $m$ is the transmitted symbol in the $k$ th frame and $x_{k, i}=X_{k, i}$ indicates the presence of the pulse at the $i$ th PPM slot. Equivalently, the received signal vector, $\mathbf{y}_{\mathbf{k}}=\left[y_{k, 1}, y_{k, 2}, \cdots, y_{k, M}\right]^{\mathrm{T}}$, is the output of the $M$ correlators at the receiver.

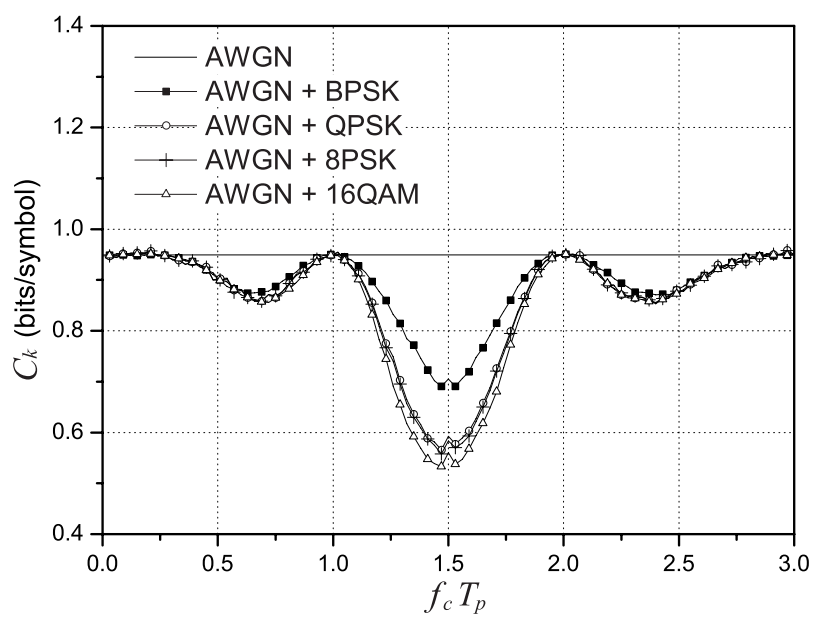

Fig. 1. Capacity of 2-PPM in AWGN channel with NBI vs. NBI frequency.

An expression of the Shannon capacity formula for $M$-ary PPM on Gaussian and Webb noise channels without interference has been presented in [14]. However, this expression cannot be used to directly compute the UWB capacity since the components of the vector $\mathbf{y}_{\mathbf{k}}$ are correlated. In order to take into account this correlation, we propose the following generic expression which, in principle, calculates the mutual information in a time interval spanning several symbols.

$$
\begin{aligned}
C_{K}= & \log _{2} M-\frac{1}{K} \int_{\mathbf{y}_{\mathbf{1}} \mathbf{y}_{\mathbf{2}} \cdots \mathbf{y}_{\mathbf{K}}} p\left(\mathbf{y}_{\mathbf{1}} \mathbf{y}_{\mathbf{2}} \cdots \mathbf{y}_{\mathbf{K}} \mid \mathbf{x}_{\mathbf{1}, \mathbf{1}} \mathbf{x}_{\mathbf{2}, \mathbf{1}} \cdots \mathbf{x}_{\mathbf{K}, \mathbf{1}}\right) \\
& \log _{2}\left[\frac{\sum_{i_{1}, i_{2}, \cdots, i_{K}=1}^{M} p\left(\mathbf{y}_{\mathbf{1}} \mathbf{y}_{\mathbf{2}} \cdots \mathbf{y}_{\mathbf{K}} \mid \mathbf{x}_{\mathbf{1}, \mathbf{i}_{\mathbf{1}}} \mathbf{x}_{\mathbf{2}, \mathbf{i}_{\mathbf{2}}} \cdots \mathbf{x}_{\mathbf{K}, \mathbf{i}_{\mathbf{K}}}\right)}{p\left(\mathbf{y}_{\mathbf{1}} \mathbf{y}_{\mathbf{2}} \cdots \mathbf{y}_{\mathbf{K}} \mid \mathbf{x}_{\mathbf{1}, \mathbf{1}} \mathbf{x}_{\mathbf{2}, \mathbf{1}} \cdots \mathbf{x}_{\mathbf{K}, \mathbf{1}}\right)}\right] \\
& d \mathbf{y}_{\mathbf{1}} d \mathbf{y}_{\mathbf{2}} \cdots d \mathbf{y}_{\mathbf{K}}
\end{aligned}
$$

where $\mathbf{y}_{1}, \mathbf{y}_{2}, \cdots, \mathbf{y}_{\mathbf{K}}$ are the received signal vectors at $K$ successive pulse intervals. Assuming perfect timing synchronization, i.e., $\tau_{j}$ and $\tau_{s}$ are known at the receiver, the conditional

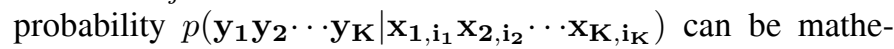
matically expressed as:

$$
\begin{aligned}
& p\left(\mathbf{y}_{\mathbf{1}} \mathbf{y}_{\mathbf{2}} \cdots \mathbf{y}_{\mathbf{K}} \mid \mathbf{x}_{\mathbf{1}, \mathbf{i}_{\mathbf{1}}} \mathbf{x}_{\mathbf{2}, \mathbf{i}_{\mathbf{2}}} \cdots \mathbf{x}_{\mathbf{K}, \mathbf{i}_{\mathbf{K}}}\right)= \\
& \frac{1}{M_{J}^{K_{J}}} \sum_{n_{1}, n_{2}, \cdots, n_{K_{J}}=1}^{M_{J}} \prod_{k=1}^{K} \prod_{m=1}^{M} N_{\mu, \sigma}\left(y_{k, m}\right)
\end{aligned}
$$

where $N_{\mu, \sigma}$ is the Gaussian probability density function (PDF) with mean $\mu=x_{k, m}+\sum_{\kappa=1}^{K_{J}} I_{k, m}^{\kappa, w_{n_{\kappa}}}$ and variance $\sigma^{2}=N_{0}$. $K_{J}$, the number of the NBI symbols included in the computation, has been chosen $K_{J} T_{J} \geq K T_{f}$ so that at least all the symbols that overlap with the $K$ UWB pulses are taken into account.

It should be noted that (8) is equivalent to the original Shannon capacity formula, with the difference that it calculates the normalized mutual information over the interval of $K$ symbols. Clearly, as $K \rightarrow \infty, C_{K}$ becomes the exact capacity. 


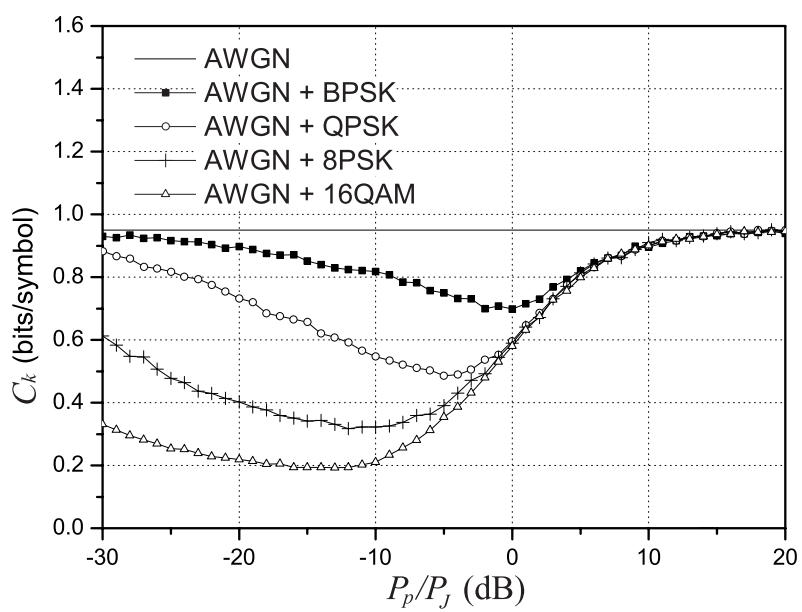

Fig. 2. Capacity of 2-PPM in AWGN channel with NBI vs. the pulse to NBI power ratio.

\section{PERFORMANCE RESULTS AND DISCUSSION}

The capacity formula (8) is evaluated with Monte-Carlo integration, as the $M$-dimensional integral makes exhaustive calculation a computationally very inefficient task [14]. For NBI, the modulations considered were binary PSK (BPSK), quadrature PSK (QPSK), 8-PSK, and 16-QAM and the UWB signal was $M$-ary PPM. Unless otherwise noted, the following parameters are used for the performance evaluation: $\beta=100, \delta_{2}-\delta_{1}=T_{p}$, $N_{p}=1, E_{p} / N_{0}=10 \mathrm{~dB}, P_{p}=P_{J}, W_{J}=\lambda=0.0077 W_{p}$, and $f_{c}=1.5 / T_{p} . W_{J}$ and $f_{c}$ are expressed as a function of $W_{p}$ and $T_{p}$, respectively, because it was observed that if $W_{J} / W_{p}$ and $f_{c} T_{p}$ are constant, the capacity evaluation results remained unchanged.

Due to the inherent complexity of (8) it turns out that the exact evaluation of $C_{K}$ for large values of $K$ is computationally extremely complex. However, extensive numerical evaluation tests have shown that, for the proposed UWB system, $C_{K}$ converges rapidly even for small values of $K$ and in practical cases, i.e., when $W_{J} \leq \lambda$, the performance results were reasonably accurate for $K>T_{J} / T_{f}$. Thus for our performance evaluation results, $K=3$ was chosen.

Fig. 1 illustrates the dependence of the UWB capacity on $f_{c}$. The not completely symmetric (with respect to $f_{c}$ ) shape of the capacity curves is due to the non-symmetric PSD of the employed UWB pulse, $q(t)$. When $f_{c}$ becomes too small or too large, the NBI signal is attenuated by the matched filter of the receiver thus diminishing its impact on capacity. Furthermore, our results verify the observation made in [15], that when $f_{c}=k / \delta$, $k \in \mathbb{N}$, the interference is completely cancelled. The minor discontinuity observed at $f_{c} T_{p}=1.5$ happens because at this frequency the interval of three frames used for the evaluation is an exact multiple of the NBI carrier period. It is also interesting to note that the QPSK and 8-PSK capacity curves in Fig. 1 are identical. This happens because for $P_{p}=P_{J}$ the UWB capacity for these two modulation schemes is equal (see Fig. 2).

Fig. 2 illustrates the performance of $C_{K}$ versus $P_{p} / P_{J}$. It is noted that the impact on capacity becomes more severe when the bits per symbol of the narrowband signal are increased. Also, these performance results are somewhat surprising since it is

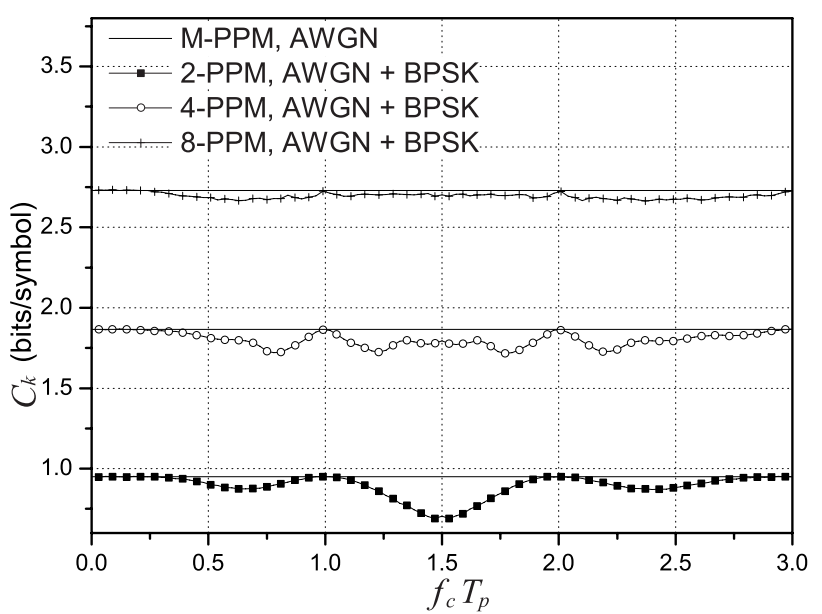

Fig. 3. Capacity of 2-, 4-, and 8-PPM in AWGN channel with NBI vs. NBI frequency.

usually expected that as $P_{J}$ increases the capacity would decrease. However, in our case, when $P_{J}$ becomes too high the degradation of the UWB capacity is reduced. This is due to the fact that the probability of incorrect decoding of the signal becomes smaller. To explain this let us consider as an example the sum of two baseband binary signals decoded by a single receiver. If both signals have similar power levels, it is reasonable to expect that the receiver cannot reliably decode them, while a significant difference in their power levels results in improved performance for both signals.

Fig. 3 presents the capacity performance of higher order $M$ ary PPM modulations, i.e., $M>2$. In this case, a significant improvement on the overall capacity performance is observed as $M$ becomes larger. Furthermore, as $M$ increases, the dependence of the capacity degradation on $f_{c}$ diminishes. Since the narrowband signal is observed by the receiver only during the reception of the UWB pulses, a higher $M$ results in a larger observation window and thus in a reduced NBI effect.

\section{CONCLUSIONS}

In this paper we have derived an expression for the capacity of a TH-UWB system in the presence of NBI. Based on this expression, we have evaluated and discussed the capacity of a TH-UWB system under such interference conditions. The obtained performance evaluation results have indicated that the effect of NBI is more severe when the narrowband signal has similar power level to the received UWB signal. Furthermore, the effect of NBI is mitigated significantly with the use of higher order PPM for the UWB signal.

\section{ACKNOWLEDGMENTS}

This paper is part of the $05 \mathrm{NON}-\mathrm{EU}-253$ research project implemented within the Framework of the "Cooperation with S\&T Institutions in non-European Countries-2005" and co-financed by National and European Community funds (25\% from the Ministry of Development-General Secretariat for Research and Technology and 75\% from the European Union-European Social Fund). 


\section{REFERENCES}

[1] S. Roy, J. R. Forester, V. S. Somayazulu, and D. G. Leeper, "Ultrawideband radio design: The promise of high-speed, short-range wireless connectivity," Proc. IEEE, vol. 92, no. 2, pp. 295-311, 2004.

[2] M. Z. Win and R. A. Scholtz, "Ultra-wide bandwidth time-hopping spreadspectrum impulse radio for wireless multiple-access communications," IEEE Trans. Commun., vol. 48, no. 4, pp. 679-691, Apr. 2000.

[3] M. Z. Win and R. A. Scholtz, "Impulse Radio: How it works," IEEE Commun. Lett., vol. 2, no. 2, 2, pp. 36-38, Feb. 1998.

[4] F. Ramirez-Mireles and R. A. Scholtz, "Multiple-access performance limits with time hopping and pulse position modulation," in Proc. IEEE Military Commun. Conf., Oct. 1998, Bedford, MA, pp. 529-533.

[5] L. Zhao and A. M. Haimovich, "Multi-user capacity of M-ary PPM ultrawideband communications," in Proc. IEEE UWBST, 2002, pp. 175-179.

[6] L. Zhao and A. M. Haimovich, "The capacity of an UWB multiple-access communications system," in Proc. IEEE ICC, vol. 3, 2002, pp. 1964-1968.

[7] G. Durisi and S. Benedetto, "Performance evaluation of TH-PPM UWB systems in the presence of multiuser interference," IEEE Commun. Lett., vol. 7, no. 5, pp. 224-226, May 2003.

[8] N. V. Kokkalis, P. T. Mathiopoulos, G. K. Karagiannidis, and C. S. Koukourlis, "Performance analysis of M-ary PPM TH-UWB systems in the presence of MUI and timing jitter," IEEE J. Sel. Areas Commun., vol. 24, no. 4, pp. 822-828, Apr. 2006.

[9] E. R. Bastidas-Puga, F. Ramirez-Mireles, and D. Munoz-Rodriguez, "On fading margin in ultrawideband communications over multipath channels," IEEE Trans. Broadcast., vol. 51, no. 3, pp. 366-370, Sept. 2005.

[10] Q. Li and L. A. Rusch, "Hybrid RAKE/multiuser receivers for UWB," in Proc. IEEE RAWCON, 2003, pp. 203-206.

[11] A. Adinoyi and H. Yanikomeroglu, "Practical capacity calculation for time-hopping ultra-wide band multiple-access communications," IEEE Commun. Lett., vol. 9, no. 7, pp. 601-603, July 2005.

[12] L. Zhao and A. M. Haimovich, "Performance of ultra-wideband communications in the presence of interference," IEEE J. Sel. Areas Commun., vol. 20, no. 9, pp. 1684-1692, Sept. 2002.

[13] L. Zhao and A. M. Haimovich, "Interference suppression in ultrawideband communications," in Proc. 35th Annual Conf. Inform. Sci. and Syst., 2001, pp. 759-763.

[14] S. Dolinar, D. Divsalar, J. Hamkins, and F. Pollara, "Capacity of pulse position modulation (PPM) on Gaussian and Webb channels," JPL TMO Progress Report 42-142, 2000.

[15] M. S. Iacobucci, M. G. Di Benedetto, and L. De Nardis, "Radio frequency interference issues in impulse radio multiple access systems," in Proc. IEEE UWBST, 2002, pp. 293-296.

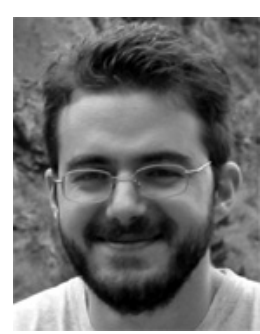

Nikos V. Kokkalis was born in Athens, Greece in 1976. He received the Electrical and Computer Engineering Diploma in 2002 from the Democritus University of Thrace, Xanthi, Greece, where he continues his studies as a Ph.D. student. His thesis is being conducted in collaboration with Institute for Space Applications and Remote Sensing (ISARS) of the National Observatory of Athens (NOA). His research interests are focused on ultra wide band communications, but also include satellite communications, cellular network optimization, forward error correction

and network protocols.

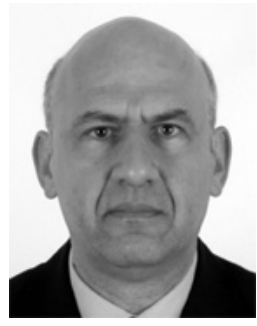

P. Takis Mathiopoulos is currently director of research at the Institute for Space Applications and Remote Sensing (ISARS) of the National Observatory of Athens (NOA), where he has established the Wireless Communications Research Group. As ISARS director, he has lead the Institute to a significant expansion, R\&D growth, and international scientific recognition. For these achievements, ISARS has been selected as one of the national Centers of Excellence for the years 20052008. Before joining ISARS, he worked in the early $80 \mathrm{~s}$ at Raytheon Canada Ltd. in the areas of airnavigational and satellite communications. In the late 80 s, he joined the Department of Electrical and Computer Engineering (ECE) of the University of British Columbia (UBC), where he was a faculty member for 14 years last holding the rank of full professor. Maintaining his ties with academia, he is an adjunct professor of ECE at UBC and also is teaching part-time at the Department of Informatics and Telecommunications, University of Athens. Over the years, Prof. Mathiopoulos has supervised university and industry based R\&D groups and has successfully acted as technical manager for large Canadian and European R\&D projects. He has also supervised the theses of 25 graduate students. His research contributions include original research work in the areas of optimal communications over fading channels, channel characterization and measurements, advanced coding techniques, including turbo-codes, diversity and synchronization, HDTV, neural networks, smart antennas, UMTS and S-UMTS, software radios, MIMOs, and UWB. He has published, together with Prof. D.Makrakis of the University of Ottawa, a paper in GLOBECOM 89 establishing for the first time in the open technical literature the link between MLSE and multiple differential detection (also known as multi-symbol differential detection) for the AWGN and fading channels. His publication record includes more then 150 papers in journals and international conference proceedings, about 50 of which have been published in IEEE journals. He has been on the editorial board of many scientific journals, including the IEEE Transactions on Communications from 1993-2005 and Journal of Communications and Networks. He has regularly acted as a consultant for several governmental and private organizations. Since 1993, he has served on a regular basis as scientific advisor and technical expert for the European Commission (EC) for the ACTS and IST programs. In this capacity, he has been appointed by the EC in numerous high level advisory, evaluation and auditing panels in the technical areas of telecommunications, information technology, and electronic commerce and publishing. He has been a member of the TPC of more than 50 international conferences including TPC vice chair for the IEEE VTC2006-S and the IEEE VTC2008-F. Prof. Mathiopoulos has delivered numerous invited presentations, including plenary lectures, and has taught many short courses all over the world.

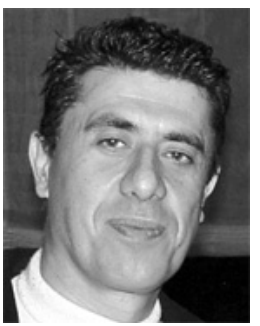

George K. Karagiannidis was born in Pithagorion, Samos Island, Greece. He received the University and Ph.D. degrees in electrical engineering from the University of Patras, Patras, Greece, in 1987 and 1999, respectively. From 2000 to 2004, he was a Senior Researcher at the Institute for Space Applications and Remote Sensing, National Observatory of Athens, Greece. In June 2004, he joined Aristotle University of Thessaloniki, Thessaloniki, Greece, where he is currently an Assistant Professor in the Electrical and Computer Engineering Department. His current research interests include wireless communication theory, digital communications over fading channels, cooperative diversity systems, cognitive radio, satellite communications, and wireless optical communications. He is the author or coauthor of more than 80 technical papers published in scientific journals and presented at international conferences. He is also a coauthor of two chapters in books and a coauthor of the Greek edition of a book on mobile communications. He serves on the editorial board of the EURASIP Journal on Wireless Communications and Networking. Dr. Karagiannidis has been a member of Technical Program Committees for several IEEE conferences. He is a member of the editorial boards of the IEEE Transactions on Communications and the IEEE Communications Letters. He is co-recipient of the Best Paper Award of the Wireless Communications Symposium (WCS) in IEEE International Conference on Communications (ICC' 07), Glasgow, U.K., June 2007. He is a full member of Sigma Xi.

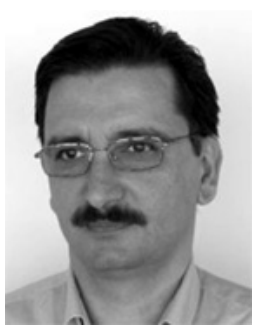

Christos S. Koukourlis was born in Kavala, Greece, on August 13, 1957. He received the Electrical Engineering Diploma in 1981 and the Ph.D degree in Electrical Engineering in 1990 both from the Democritus University of Thrace, Xanthi, Greece. He currently serves as an Associate Professor in the same Department working on digital modulation and high spectral efficiency techniques. His interests also include Direct Digital Synthesis (DDS) methods, Communications Networks and Fleet Management (GPS based) Systems over GSM network. 